\title{
Optimization Model of Process Parameters for Full-Plate Long-Roll Straightening considering Equipment Bounce
}

\author{
Xiaoyu Zhu, Peisheng Han, Xiaoxiao Liu, and XiaoGang Wang (iD \\ Engineering Research Center Heavy Machinery Ministry of Education, Taiyuan University of Science and Technology, \\ Shanxi Taiyuan 030024, China \\ Correspondence should be addressed to XiaoGang Wang; wxg@tyust.edu.cn
}

Received 20 November 2020; Revised 26 January 2021; Accepted 3 February 2021; Published 17 February 2021

Academic Editor: Rossana Dimitri

Copyright (c) 2021 Xiaoyu Zhu et al. This is an open access article distributed under the Creative Commons Attribution License, which permits unrestricted use, distribution, and reproduction in any medium, provided the original work is properly cited.

Roll straightening is an important process link in sheet production. Due to the step change of the straightening force during the straightening bite and the tail flick, the whole-plate length straightening and pressing process changes will affect the straightening effect. Based on the solution of the curvature integral straightening process, this paper introduces the stiffness coefficient of the straightening equipment. Through $\mathrm{C}$ language programming, the iterative solution has been realized, and the process parameters of the dynamic change of the straightening reduction have been obtained. The enumeration method was used to establish a calculation method to optimize the entire board-length straightening process. The laboratory comparison of the 11-roll straightening machine proves that the straightening process optimization model meets the application requirements.

\section{Introduction}

Roll straightening, as an important process step in sheet production, directly determines product quality. The setting and optimization of process parameters have been the focus of research by domestic and foreign scholars due to the nonlinearity of the metal material and the stiffness of the straightening equipment. At present, a lot of research has been conducted at home and abroad on the theory of straightening and the setting of process parameters. Cui [1] deduced the calculation method of each parameter in the straightening process by studying the continuous simply supported beam model. He [2] performed an analysis by reinforcing the material and concluded that the large deformation strategy residual-curvature control is better than the small deformation strategy. Grüber and Hirt [3] performed the analysis of curvature change and residual stress during the straightening process based on the finite element method. Li et al. [4] simulated the tension-leveling process using two-dimensional and three-dimensional frames. The results showed that the tension-leveling process should be simulated in three dimensions. Cui and Shi [5] investigated the effect of the grid interval on the residual curvature of longitudinal profiled plates. Liu et al. [6] studied the evolution of stress in a continuous bending process, and the effect of the strain-hardening model was studied. Despite being widely available, these models fail to satisfy the everincreasing requirement for flatness because the models do not fully consider the contact state between the plate and rollers. Fumio [7] obtained that the contact point is located in front of the top point of the straightening roller by experimental verification of the plate strip, but the location is extremely difficult to determine. Tani et al. [8] further verified the contact point between the plate and the straightening roll, which is not directly below the straightening roll, by the finite element analysis method and experiments. Kado and Maeda [9] proposed a new analytical model of continuous curvature for the solution of residual curvature. Goshi et al. [10] performed curvature integration by the straightening process and solved it. Xue et al. [11-13] suggested that the contact angle has a cumulative effect throughout the straightening process, and this cumulative 
effect hurts the residual curvature, which was verified by numerical simulation for calculation. Liu et al. [14-16] used numerical algorithms to solve for the curvature and concluded that the original curvature has a large effect on the contact angle of the first few rolls. Yin et al. [17] studied the section steel-leveling process on a flexible roller system based on springback theory. They believe that the large and small deformation-leveling strategy definition is helpful to set reasonable roller intermeshes according to a different plate. Also, they improve the section steel-leveling quality by adjusting the last rollers. Zhang et al. [18] studied the model and finite element analysis of the steel strip tensioning, and the leveling process was carried out to corroborate the straightening process. All the above studies were based on the premise of a straightening steady state.

During the straightening process, the strapping and tail flicking off the strip will cause a step change in the straightening force, resulting in different deformations of the equipment and affecting the change of the straightening process parameters. Japanese scholars $[19,20]$ studied the impact of bounce on straightening but did not give a feasible straightening process plan.

In this paper, based on the curvature integral solution process, the inclined straightening model is derived, as well as the optimization method of the bounce straightening process model, and the model is iteratively calculated using C language programming. A laboratory eleven-roll straightening machine verified it.

\section{A Curvature Integral Model of the Straightening Process}

In this paper, the plate straightening process is conducted by using an eleven-roller leveler, as shown in Figure 1. Five rollers are mounted on the upper support and the others on the lower support. The lower support is fixed on the frame, while the upper support is connected to the four hydraulic cylinders. The hydraulic cylinders set the roller gaps in advance. The plate is bent in an alternate direction by the rollers when it goes through the leveler. It is forced into elastic-plastic deformation due to the bidirectional bending exerted by staggered gaps. Therefore, the leveling process can be considered a continuous alternating bending and reverse bending process. The plate defects including flatness imperfections can be eliminated in the leveling process. Use the pressure sensor to observe the straightening force.

2.1. Analysis of Curvature Integral Theory. The straightening of the plate is a process of repeated bending; curvature is a physical quantity that describes the degree of curvature of an object in space. It vividly expresses the entire bending state of the plate during the straightening process, so it can construct a straightening calculation model based on the curvature of the research condition [21, 22], as shown in Figure 2. A few explanations for the calculation model are provided.

(i) The contact points of the $i$ and $i+1$ rollers are $A\left(x_{i}, z_{i}\right)$ and $B\left(x_{i+1}, z_{i+1}\right)$ (ii) The distance between the contact points $A$ and $B$ of the $i$ and $i+1$ roller is $L_{i}$

(iii) The angle $\left(\theta_{x}\right)$ between the tangent from $A$ to $B$ and the $x$-axis greater than $90^{\circ}$ is " + " and less than is "-"

(iv) $\delta_{i}$ is the reduction of the upper roller $i$ along the $z$-axis

Through the above points, the mechanical conditions satisfied by any point $c$ on the straightening interval are as follows:

$$
\begin{aligned}
M_{i} & =\int_{-B / 2}^{B / 2} \int_{-H / 2}^{H / 2} \sigma_{z}(x) d y d z \\
M_{x} & =M_{i}+\frac{\left(M_{i+1}-M_{i}\right) x}{L_{i}} .
\end{aligned}
$$

The relationship between the curvature $k_{x}$ of the neutral layer of the sheet section and the bending deflection $y$ is as the following equation:

$$
\frac{1}{\rho_{x}}=k_{x}=\frac{d^{2} y}{d x^{2}}=\frac{M_{x}}{\mathrm{EI}}
$$

where $M_{x}$ is the bending moment at any part of the section, $\sigma_{z}(x)$ is the bending stress distribution of the sheet section, $E$ is Young's modulus, and $I$ is the inertia moment of the geometric section.

The contact angles $\theta_{x}$ and down feed $z_{x}$ can be calculated by the first and second integral of curvature $k_{x}$ :

$$
\begin{aligned}
& \theta_{x}=\int_{0}^{x} k_{x} d x+C_{1}, \\
& z_{x}=\int_{0}^{X} \theta_{x} d x+C_{2}=\int_{0}^{X} \int_{0}^{x} k_{x} d x d X+C_{1}(X-0)+C_{2} .
\end{aligned}
$$

When point $C$ moves to point $A$ and $B$ in Figure 2, equations (4) and (5) can be further derived as follows:

$$
\begin{aligned}
& \mp \theta_{i+1}=\int_{0}^{L_{i}} k_{x} d x \pm \theta_{i}, \\
& \mp z_{i+1}=\int_{0}^{L_{i}} \int_{0}^{L_{i}} k_{x} d x d X \pm \theta_{i},
\end{aligned}
$$

where the sign-in equations (6) and (7) depend on the roller ID $i$. If the $i$ roller is the upper roller, the sign-in equation (6) is "+," while it is "-" in equation (7). Otherwise, the sign-in equation (6) is "-," while it is "+" in equation (7).

In Figure 2, the geometric relationship can be obtained:

$$
\begin{aligned}
z_{i+1}= & \delta_{i+1}-R\left(1-\cos \theta_{i}\right)-R\left(1-\cos \theta_{i+1}\right), \\
& \mp\left(\delta_{i+1}-R\left(1-\cos \theta_{i}\right)-R\left(1-\cos \theta_{i+1}\right)\right) \\
= & \int_{0}^{L_{i}} \int_{0}^{L_{i}} k_{x} d x d X \pm \theta_{i},
\end{aligned}
$$




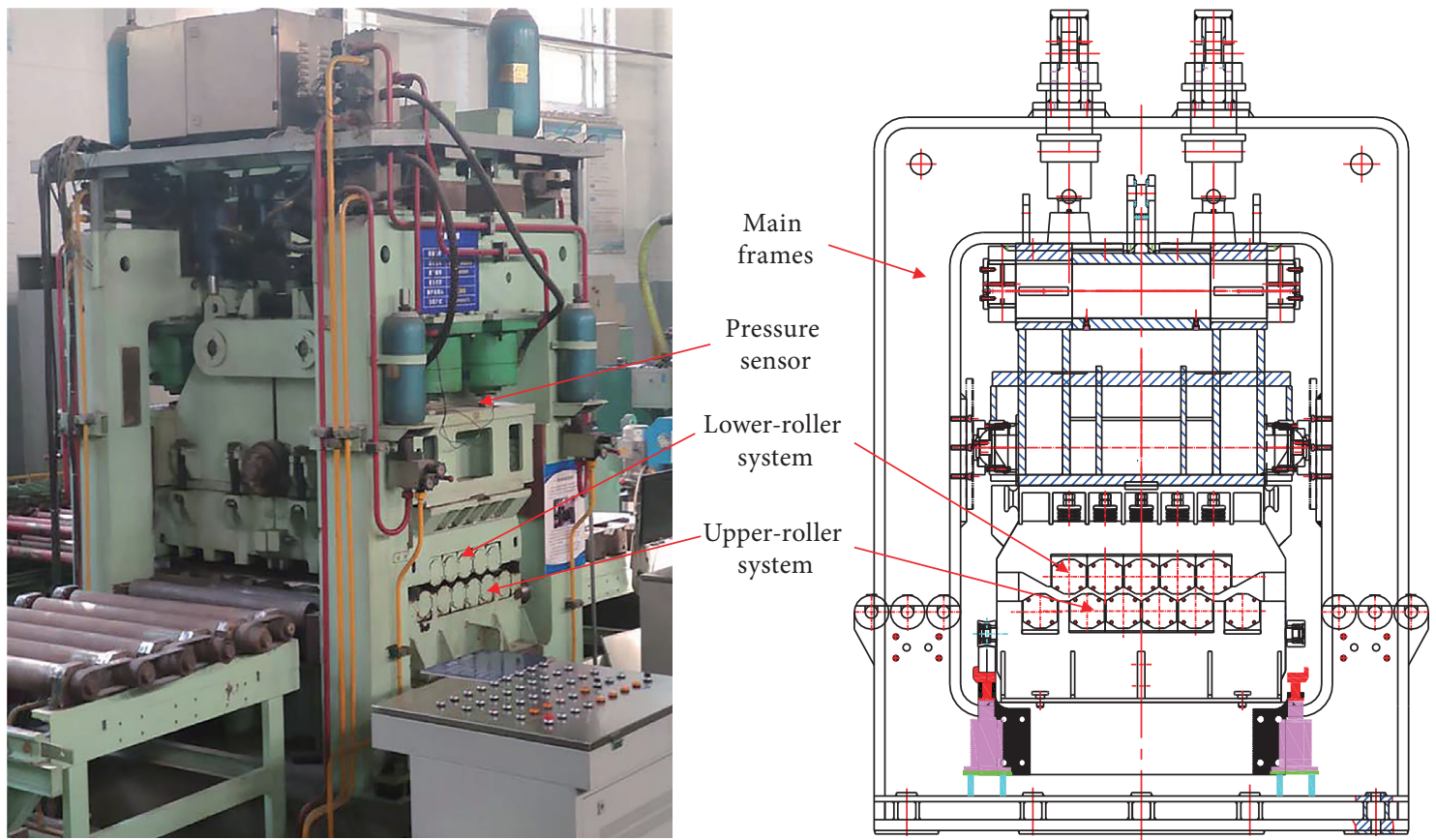

FIGURE 1: Straightening equipment and its structure.

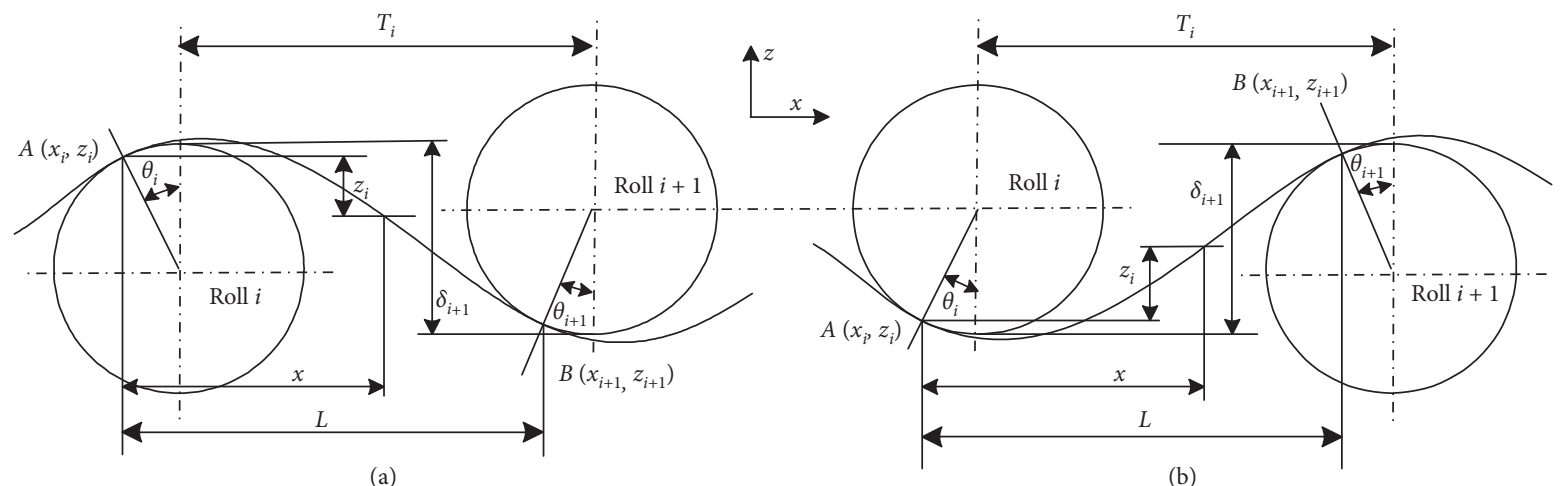

Figure 2: Schematic diagram of the curvature integral-method model construction.

where $R$ is the radius of the straightening roll.

In the curvature solution of the whole straightening process, the straightening interval equations are constructed according to the relationship between the contact angle, the reduction amount, and the reverse bending rate of each straightening interval, as shown in equations (8) and (9). Suppose the number of straightening rollers is $n$, then a nonlinear equation group consisting of $2 \times(n-1)$ nonlinear equations can be constructed, in which there are $2 \times(n-1)$ unknowns, $\theta_{1}-\theta_{n} k_{2}-$ $k_{n-1}$. The unknown number is equal to the number of equations, and the residual curvature, contact angle, and inverse bending rate can be calculated theoretically.
2.2. Straightening Process. The plate straightening process can be divided into three stages: biting, continuous straightening, and tail flicking. The straightening force depends on different straightening stages, as shown in Figure 3.

At the beginning of the biting phase, the head of the plate gradually enters the straightening machine. As time $t$ increases, at $t_{1}$, the straightening from the three rollers increases to the eleven-rollers' straightening, reaching a stable stage. During this process, the straightening force increases stepwise. Under the action of the straightening force, the roll gap is continuously increasing relative to the 


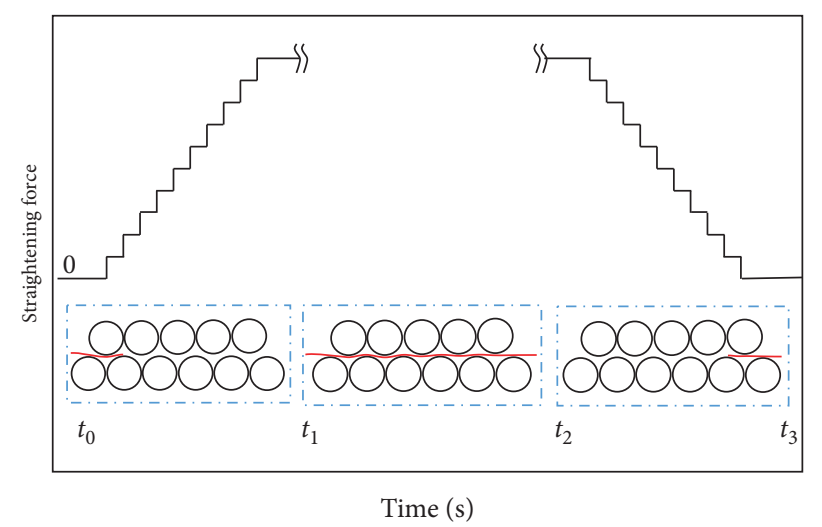

FIgURE 3: Variation of straightening force during the straightening process.

initial roll gap, and it tends to be stable after the plate passes through the roll system. In the tail-flicking phase, starting at $t_{2}$, the plates leave in sequence, and eleven rollers reduce the number of rollers acting on the plate. At $t_{3}$, the straightening is completed. In this process, the straightening force decreases successively, the bounce of the equipment decreases, and the roll gap gradually returns to the initial setting state.

In the whole process of sheet straightening, suppose the straightening force is $P_{i}$, the reduction is $z_{i}$, and the real reduction is $z_{i}$, the relationship between the three is as follows:

$$
\begin{aligned}
& \Delta_{i}=\frac{P_{i}}{K}, \\
& z_{i}=z_{i}-\Delta_{i} .
\end{aligned}
$$

First of all, from the different stages of plate biting, continuous and stable straightening, and tail-flicking, according to the different number of straightening and continuous bending, three-rollers' straightening to elevenrollers' straightening and different curvature integral equations are constructed. In this process, consider the influence of the changes in the straightening state during the three rollers' straightening to the eleven rollers over the straightening process on the calculation of the straightening process. Secondly, through the result of curvature integral calculation, the straightening force and equipment bounce at different moments are further calculated. Recalculate the calculated device bounce into the depression parameter. Finally, repeat this process to keep the straightening force and equipment bounce in balance. The calculation process is shown in Figure 4.

\section{Straightening Process Optimization Method}

The residual curvature after straightening is the main indicator of straightening quality. For the residual curvature of each stage of eleven-rollers' straightening, it depends on the first and last roll-reduction amount $z_{2}$ and $z_{10}$ :

$$
C_{C}=\left[z_{2}, z_{10}\right]
$$

Therefore, the problem of the straightening process is attributed to the maximum residual curvature in the total plate length required for different $z_{2}$ and $z_{10}$ and the minimum residual curvature in the feasible region of $z_{2}$ and $z_{10}$.

The optimization model is as follows:

(1) The design variables are $z_{2}$ and $z_{10}$

(2) The objective synthesis function is as follows:

$$
C_{C \text { optimal }}=\min \left[\max \left(C_{C(i, j) \text { biting }}, C_{C(i, j) \text { continuous }}, C_{C(i, j) \text { tail }}\right)\right] \text {. }
$$

According to the characteristics of the straightening process setting, the optimization method is solved by the enumeration method, assuming that the first reduction can take the value of $n$, and the value range is $k_{w}=(1-5) k_{t}$. The end reduction can take the value of $m$, and the value range is $k_{w}=(0-1) k_{t}$; there will be a total of $n \times m$ process parameters. According to the actual straightening situation, in the head, middle, and tail, the bounces produced are different, corresponding to $3 \times n \times m$ residual curvature values, and the optimal straightening process plan is calculated and judged.

\section{Calculation Results and Experimental Analysis}

The main technical parameters of the straightening equipment and the mechanical properties of the straightened sheet are shown in Table 1.

4.1. Calculation Results. According to the calculation model in this paper, the calculation is performed on the plate with length $\times$ width $\times$ height of $1000 \mathrm{~mm} \times 350 \mathrm{~mm} \times 4 \sim 6 \mathrm{~mm}$, assuming that the initial curvature is 0 , the material is isotropic, and there is no residual stress distribution. The 


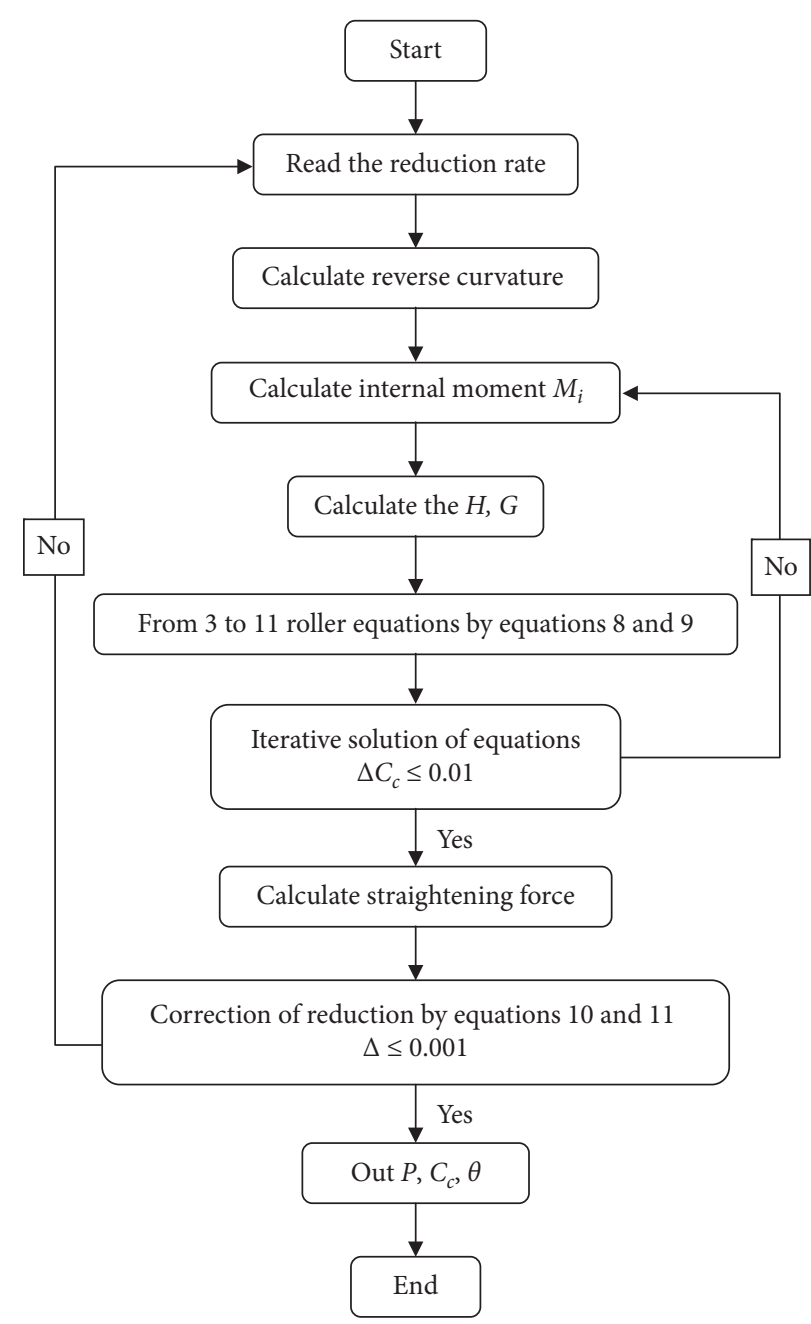

FIgUre 4: Calculation flow of whole-plate straightening.

TABLE 1: Mechanical property parameters of straightening equipment and straightening plate.

\begin{tabular}{lccc}
\hline Abbreviation & Unit & Meaning & Parameter value \\
\hline$N$ & - & Number of work rollers & 11 \\
$D$ & $\mathrm{~m}$ & Roller diameter & 0.095 \\
$T$ & $\mathrm{~m}$ & Roller space & 0.1 \\
$K$ & $\mathrm{~m}$ & Machine stiffness coefficient & $2 \times 10^{8}$ \\
$\sigma_{s}$ & $\mathrm{~Pa}$ & Yield stress & $2.35 \times 10^{8}$ \\
$E$ & $\mathrm{~Pa}$ & Young's modulus & $2.1 \times 10^{11}$ \\
$\mu$ & - & Poisson's ratio & 0.3 \\
$L$ & $\mathrm{~m}$ & Plate length & 1 \\
$B$ & $\mathrm{~m}$ & Plate width & 0.35 \\
$h$ & $\mathrm{~m}$ & Plate height & $0.004 \sim 0.006$ \\
\hline
\end{tabular}

calculation results of the head, continuous straightening, and residual curvature of the tail are shown in Figures 5-7.

As shown in Figures 5-7, in the stable stage, the result of the residual curvature set by the traditional reduction is significantly greater than the calculation with the bounce setting, and the single-roll reduction adjustment is close to the overall reduction setting. During the biting process, as shown in Figures 5(a)-7(a), the dynamic single-roll setting calculation is used for the initial biting, which is closer to the traditional reduction calculation result. As the number of biting rolls 


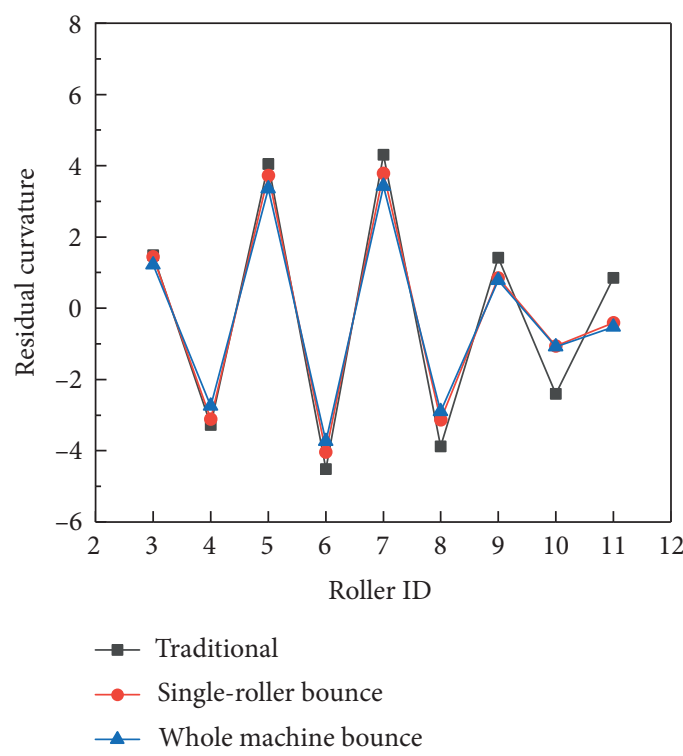

(a)

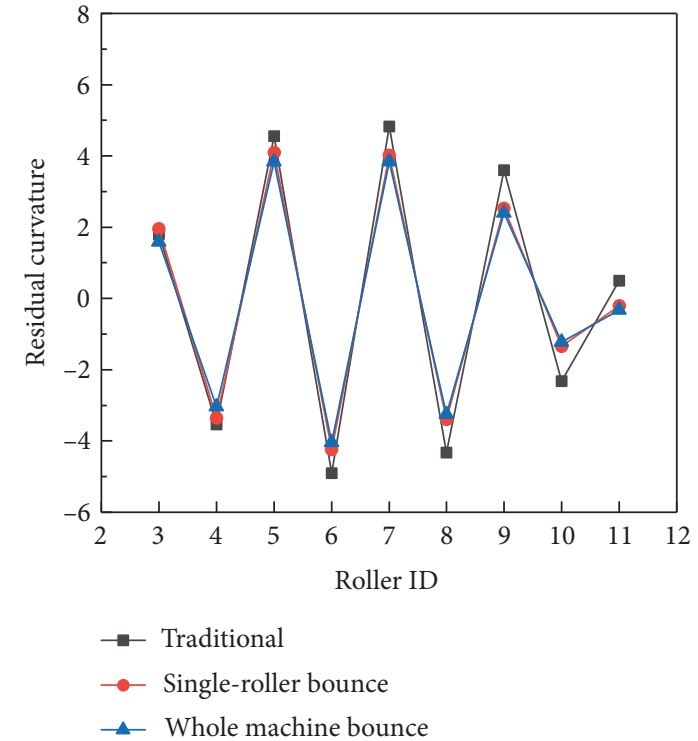

(b)

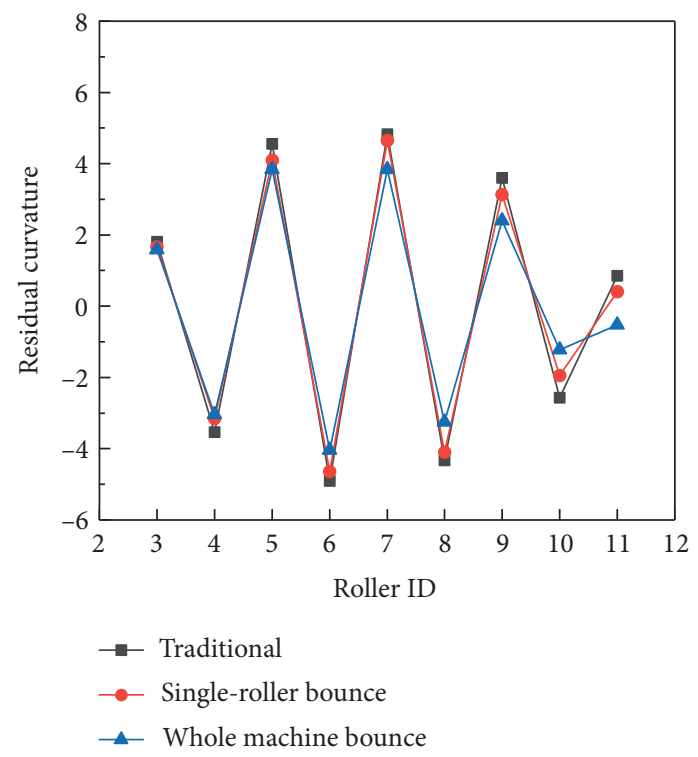

(c)

Figure 5: Variation of residual curvature during straightening of $4 \mathrm{~mm}$ plate thickness: (a) biting process; (b) continuous straightening process; (c) tail-flick process.

increases, the single-roller dynamic reduction setting is closer to the calculation of the overall bounce-reduction setting. As the plate is completely bitten, the overall frame force becomes stable and enters a stable stage, which is also in line with Figures' 5(b)-7(b) happening. During the tail-flicking process, the plate gradually leaves the straightening machine. At this time, the one-way total straightening force is reduced, the reduction gradually returns to the traditional setting, and the residual curvature returns from the overall reduction adjustment to the traditional reduction setting; the result is closer to using traditional calculations. Therefore, the variability of the reduction during this change process causes the curvature of the final straightened sheet to be inconsistent. This is consistent with the actual situation.

It can be seen from Figures 5-7 that as the thickness of the plate increases, the impact of bounce on the straightening process gradually increases. Because the rigidity coefficient of the equipment remains unchanged, the thickness 


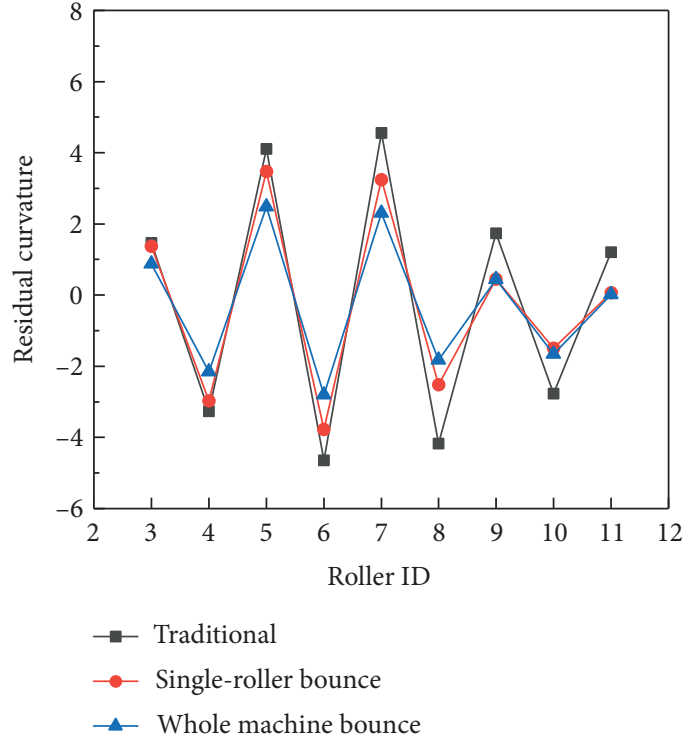

(a)

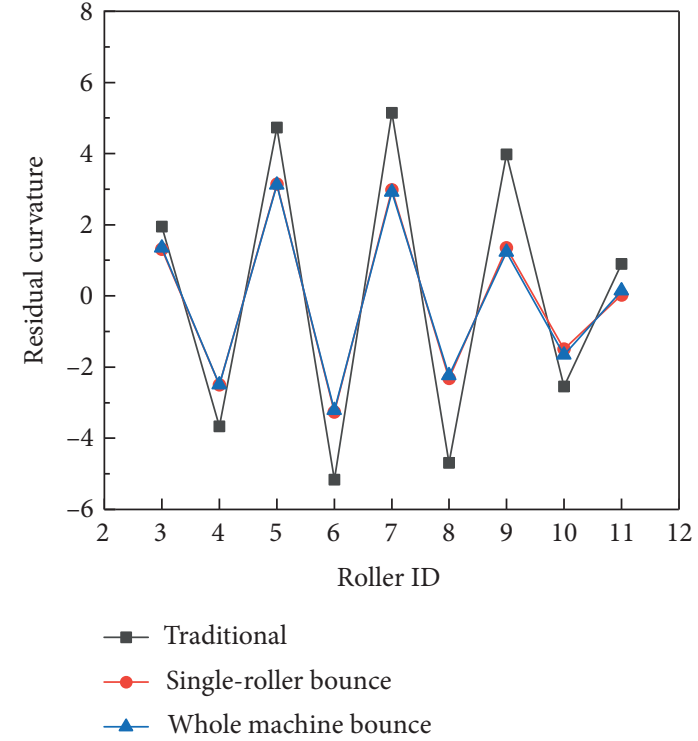

(b)

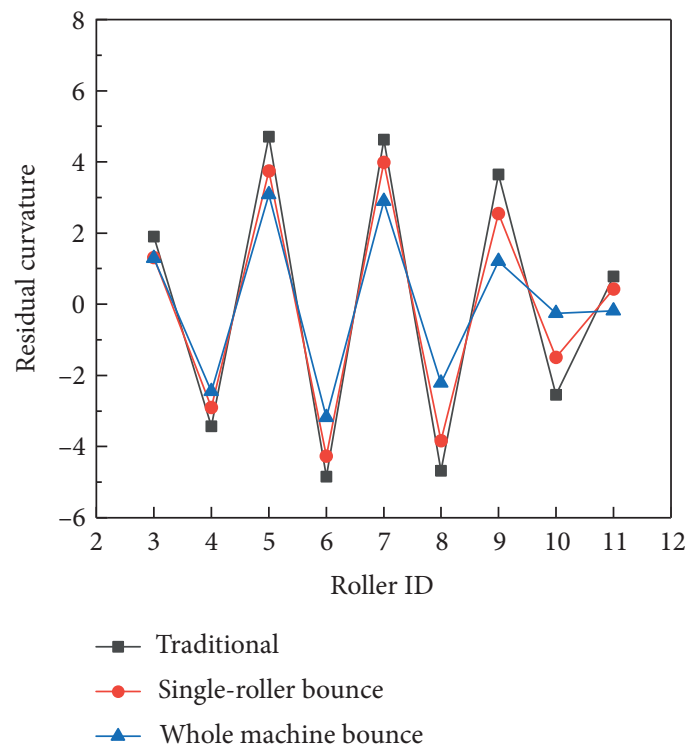

(c)

Figure 6: Variation of residual curvature during straightening of $5 \mathrm{~mm}$ plate thickness: (a) biting process; (b) continuous straightening process; (c) tail-flick process.

increases when the straightening equipment remains unchanged, increasing the one-way straightening force and causing a larger bounce factor. At the same time, the reduction of the thick plate is relatively small, so it is more likely to cause changes in the straightening process.
4.2. Experimental Analysis. Straightening is carried out for different sheet thicknesses. The specifications and process parameters are shown in Table 2. The residual curvature value after straightening is between 0.3 and 0.5 , and the one-way straightening force error is within $10 \%$. The model 


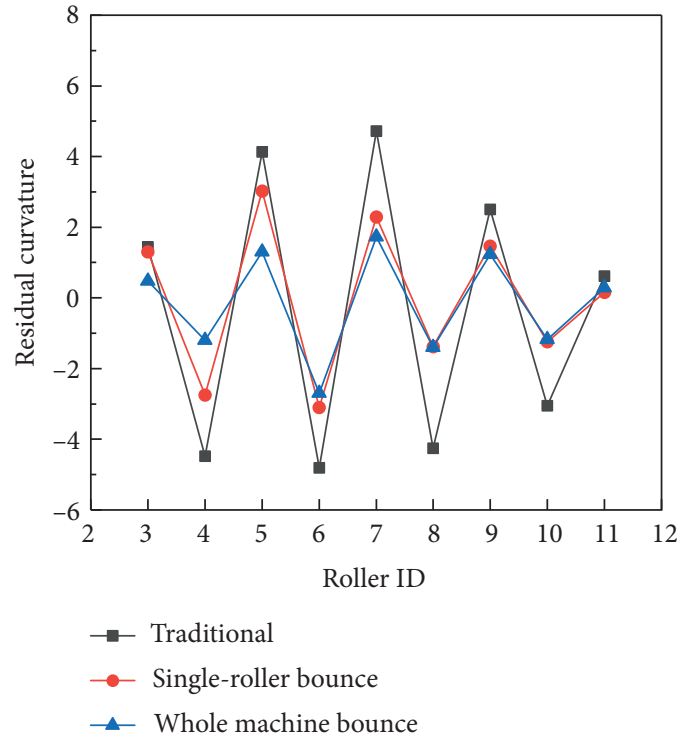

(a)

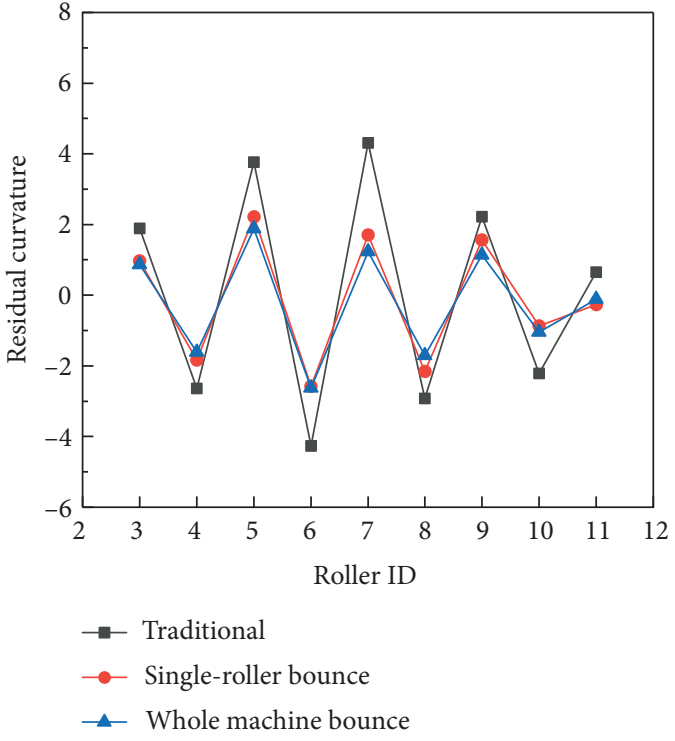

(b)

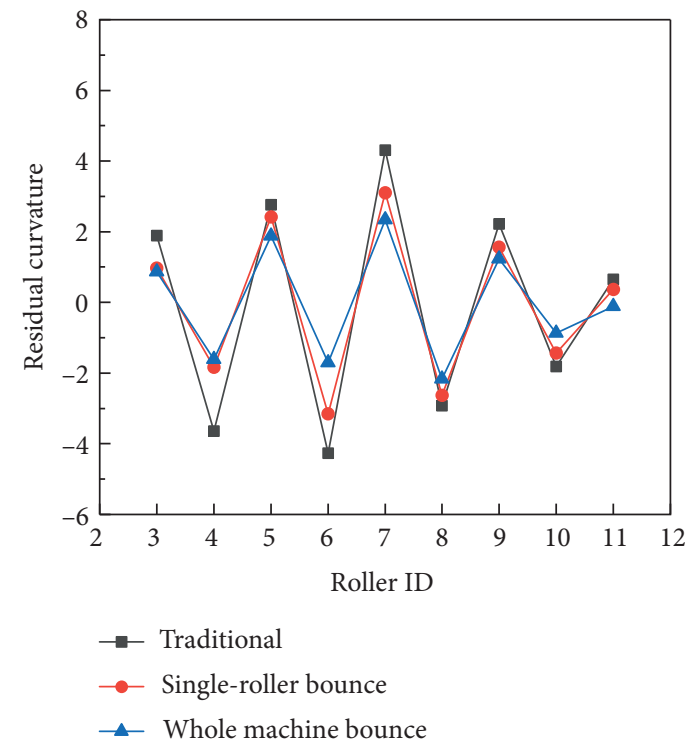

(c)

FIGURE 7: Variation of residual curvature during straightening of $6 \mathrm{~mm}$ plate thickness: (a) biting process; (b) continuous straightening process; (c) tail-flick process.

TABLE 2: Comparison of calculation and experimental data.

\begin{tabular}{lcccccccc}
\hline & \multicolumn{2}{c}{$\begin{array}{c}\text { Reduction } \\
\text { Plate height }(\mathrm{mm})\end{array}$} & \multicolumn{3}{c}{ Straightening force $(\mathrm{kN})$} & \multicolumn{3}{c}{ Residual curvature } \\
& $z_{2}$ & $z_{10}$ & Calculated & Experimental & Error (\%) & Calculated & Experimental & Error (\%) \\
\hline 4 & 1.1 & -0.1 & 96.82 & 89.65 & 7.99 & -0.40 & 0.39 \\
5 & 0.8 & -0.2 & 151.30 & 146.25 & 3.45 & 0.42 & 0.43 & -3.89 \\
6 & 0.6 & -0.2 & 217.91 & 208.91 & 4.30 & 0.45 & 0.42 & 6.38 \\
\hline
\end{tabular}


algorithm is consistent with the live test value. The calculated value of the straightening force model is smaller than the measured data. The reason may be as follows: in the model establishment, factors such as material work hardening were not considered.

\section{Conclusion}

In this paper, the device stiffness factor is introduced to analyze the curvature of the full-plate length straightening process. Based on the above calculations and experimental results, the following conclusions are drawn:

(1) Established a calculation model considering the residual curvature of the whole plate after the equipment bounced and straightened.

(2) Established a full-plate long-roll straightening process parameter optimization model considering equipment bounce to meet the application requirements of straightening process parameter optimization.

(3) Calculated through examples, during the biting process, the impact of the bounce factor gradually increases, and during the continuous straightening process, the impact tends to be stable and gradually decreases in the final flick stage. Besides, with the increase of plate thickness, the change of bounce to the straightening process parameters increases.

\section{Data Availability}

The processed optimization model required to reproduce these findings cannot be shared at this time as the data also form part of an ongoing research in the coming academic year.

\section{Conflicts of Interest}

The authors declare that they have no conflicts of interest.

\section{Acknowledgments}

This work was supported by the National Natural Science Foundation of China Project (51675362).

\section{References}

[1] F. Cui, Straightening Principle and Straightening Machine, Beijing Metallurgical Industry Press, Beijing, China, 2002.

[2] A. He, "Research on roller straightening mechanical behavior of plastic hardening material," Journal of Mechanical Engineering, vol. 52, no. 1, 2016.

[3] M. Grüber and G. Hirt, "A strategy for the controlled setting of flatness and residual stress distribution in sheet metals via roller levelling," Procedia Engineering, vol. 207, pp. 1332-1337, 2017.

[4] S.-z. Li, Y.-d. Yin, J. Xu, J.-m. Hou, and J. Yoon, "Numerical simulation of continuous tension leveling process of thin strip steel and its application," Journal of Iron and Steel Research International, vol. 14, no. 6, pp. 8-13, 2007.
[5] L. Cui and Q. Q. Shi, "Residual curvature of longitudinal profile plate roller in leveling process," Journal of Iron and Steel Research(International), vol. 10, pp. 26-30, 2013.

[6] D. Liu, A. R. He, H. B. Wang et al., "Reverse bending research of leveling on plastic hardening material," Journal of Mechanical Engineering, vol. 51, no. 8, pp. 76-82, 2015.

[7] H. Fumio, "Problems of correction technology," Research on Machinery, vol. 31, no. 8, pp. 13-18, 1979.

[8] T. Tani, K. Oe, and K. Maeda, "Analysis of stress during 3D FEM thick plate correction," CAMP-ISIJ, vol. 12, p. 1092, 1999.

[9] K. Kado and R. Maeda, "Analysis of curvature during the straightening process of roller straighteners," Plasticity and Machining, vol. 34, pp. 388-481, 1993.

[10] B. Goshi, Matsumoto, and Ogawa, "Effect of stress-strain relationship on the curvature of straightened material during roller straightening," Plasticity and Machining, vol. 43, no. 496, pp. 79-83, 2002.

[11] J. A. Xue, Theoretical Analysis of Medium-Thick Plate Straightening Process and its Control System, Northeastern University, Beijing, China, 2009.

[12] J. A. Xue, X. L. Hu, and X. Liu, "Mathematical model of elastic-plastic bending for roller leveling," Journal of Iran and Steel Research, vol. 11, pp. 33-36, 2008.

[13] J. A. Xue, L. Cui, and X. L. Hu, Analysis of Contact Angle and Curvature during Roller Leveling Process, Chins Metallurgy, Beijing, China, 2009.

[14] Z. F. Liu, Study of Leveling Theory Based on Curvature Integration Method for Plates, College of Mechanical Engineering of Chongqing University, Beijing, China, 2014.

[15] Y. Q. Wang and Z. F. Liu, "Curvature analysis for plate during hot leveling," Iron \& Steel, vol. 47, no. 1, pp. 51-54, 2012.

[16] Z. F. Liu, Y. X. Luo, Y. Q. Wang, T. W. Liao, and M. Deng, "An analytical study of optimal roller intermeshes for the plate leveling process," 2020.

[17] J. Yin, J. Zhao, S.-Y. Wang, X.-S. Wan, and Y.-L. Li, “Principle of multi-roller straightening process and quantitative resolutions of straightening strategies," Journal of Iron and Steel Research International, vol. 21, no. 9, pp. 823-829, 2014.

[18] Q. Zhang, S. Zhou, X. F. Zhang et al., "Analytic modeling and corroborating by FEM of tension leveling process of thin buckled steel strip," Journal of Mechanical Engineering, vol. 51, no. 2, pp. 49-57, 2015.

[19] H. Takeshi and M. Hiromi, "Roller straightening analysis considering the rigidity of the straightening machine (1) -rigidity identification method of the roller straightening machine- (2000 spring lecture on plastic working)," 2000.

[20] H. Takeshi and M. Hiromi, "Roller straightening analysis considering the rigidity of the straightening machine (2) -Proposal of analysis method and comparison with experimental results (51st Joint Lecture on Plastic Machining)," 2000.

[21] J. Araki and N. Yamamoto, "Research Bulletin: effect of fulcrum arrangement of orthodontic machine on final curvature of orthodontic material," Manufacturing Research, vol. 25, 1973.

[22] J. Araki, "Effect of the number of fulcrums and fulcrum arrangement of the straightening machine on the final curvature of the straightening material (one analysis method on the deformation process of the straightening material-4)," Plasticity and Machining, vol. 15, 1974. 